\title{
Mass gap and finite-size effects in finite temperature SU (2) lattice gauge theory th
}

\author{
J. Engels and V.K. Mitrjushkin ${ }^{1}$ \\ Fakultät für Physik, Universität Bielefeld, W-4800 Bielefeld, FRG
}

Received 14 January 1992; revised manuscript received 1 March 1992

\begin{abstract}
This letter is devoted to the investigation of the point-point Polyakov loop correlators in SU(2) lattice gauge theory on $4 N_{\mathrm{s}}^{3}$ lattices with $N_{\mathrm{s}}=8,12,18$ and 26 . We use an analytic expression for point-point correlators provided by the transfer matrix formalism to study the temperature dependence of the mass gap $\mu_{\mathrm{m} . \mathrm{g}}$ and the corresponding matrix element $v$ near the critical point in a finite volume. The finite-size scaling analysis of the values $\mu_{\text {m.g. }}\left(\beta, N_{\mathrm{s}}\right)$ obtained gives the possibility to extract the critical value $\beta_{c}$, the critical exponent $\nu$ and the surface tension $\alpha_{\text {s.t. }}$.
\end{abstract}

\section{Introduction}

As is well known in $\mathrm{SU}(N)$ gauge theories the confinement-deconfinement phase transition connected with the spontaneous breaking of the global $\mathrm{Z}_{N}$ symmetry occurs at some nonzero temperature $\theta_{c}[1,2]$. The numerical study of transition phenomena is a rather delicate problem because of strong finite volume effects near the transition point. On a finite lattice the symmetry can never be spontaneously broken, and the tunneling between different minima of the effective potential at $\theta>\theta_{c}$ lifts the degeneration of the vacuum in the deconfinement phase making the results sensible to the size of the lattice near $\theta_{\mathrm{c}}$. Instead of two degenerate vacua $\left|0_{+}\right\rangle$and $\left|0_{-}\right\rangle$(for the SU (2) group) one rather has on finite lattices two nondegenerate "mixed" states $\left|0_{s}\right\rangle$ and $\left|0_{a}\right\rangle$ above $\theta_{\mathrm{c}}$. In this letter we study the influence of the tunneling phenomena on the point-point Polyakov loop correlators in the SU(2) lattice gauge theory. The standard Wilson action is

$$
S_{\mathrm{W}}\left(U_{\ell}\right)=\beta \sum_{\square}\left(1-\frac{1}{2} \operatorname{Tr} U_{\square}\right),
$$

Work supported by the Deutsche Forschungsgemeinschaft under research grant En 164/2-3.

1 Permanent adress: Joint Institute for Nuclear Research, Dubna, Russia, USSR. where $\beta=4 / g_{\text {bare }}^{2}$ and $U_{\square} \in \mathrm{SU}(2)$ are plaquette variables. On a lattice $N_{\mathrm{t}} \cdot N_{\mathrm{s}}^{3}$ the temperature is defined as $\theta \equiv 1 / a N_{\mathrm{t}}$ where $a \equiv a(\beta)$ is the lattice spacing. In what follows we shall put the spacing equal to unity, measuring, therefore, all distances and energies in units of $a$ and $a^{-1}$, respectively. The average of the Polyakov loop $\mathscr{P}(\boldsymbol{x})$ is defined in a standard way:

$\langle\mathscr{P}\rangle \equiv Z^{-1} \int \prod_{\text {links }} \mathrm{d} U_{\ell} \mathscr{P}(\boldsymbol{x}) \exp \left[-S_{\mathrm{w}}\left(U_{\ell}\right)\right]$,

where

$\mathscr{P}(\boldsymbol{x}) \equiv \frac{1}{2} \operatorname{Tr}\left(\prod_{\tau=1}^{N_{\mathrm{t}}} U_{4}(\boldsymbol{x}, \tau)\right)$

and periodic boundary conditions in all directions are assumed.

In the infinite volume limit the average value of the Polyakov loop $\langle\mathscr{P}\rangle$ is zero below the critical point and differs from zero at $\theta>\theta_{\mathrm{c}}\left(\beta>\beta_{\mathrm{c}}\right)$ because of spontaneous breaking of the global $\mathrm{Z}_{2}$ symmetry. The point-point correlator $\Gamma(x)$ of the Polyakov loops defines the color averaged potential for a static qq̄ pair separated by a distance $|\boldsymbol{x}|$

$$
\Gamma(\boldsymbol{x})=\langle\mathscr{P}(\boldsymbol{x}) \mathscr{P}(\boldsymbol{0})\rangle \sim \exp \left(-\frac{1}{\theta} V_{\mathrm{q} \overline{\mathbf{q}}}(\boldsymbol{x} ; \theta)\right) .
$$


The temperature dependence of this potential is of considerable interest for a quantitative understanding of colour screening and its possible relation to the deconfinement transition. One expects that below the phase transition point $\theta_{\mathrm{c}}$ (or $\beta_{\mathrm{c}}$ ) the potential is linearly rising at large distances $(1 / \theta) V_{\mathrm{q} \tilde{\mathrm{q}}}(r ; \theta)=$ $\mu_{\mathrm{m} . \mathrm{g} .}(\theta) r+\ldots$ where $\mu_{\mathrm{m} . \mathrm{g} .}$ is the so-called mass gap, while above the phase transition point $\mu_{\mathrm{m} . \mathrm{g} .}=0$ and Debye screening of static color charges presumably takes place.

For the investigation of the long distance properties of the plasma phase the correct functional form of the potential $V_{\mathrm{q} \bar{q}}(x ; \theta)$ is of principal importance, especially in view of speculations about the possible breakdown of simple Debye screened Coulomb behaviour [3]. The usual choice for the parametrization of $\mathrm{V}_{\mathrm{q} \overline{\mathrm{q}}}(\boldsymbol{x} ; \theta)$ above the phase transition point is

$$
\begin{aligned}
& V_{\mathrm{q} \overline{\mathrm{q}}}(r ; \theta)=c(\theta)\left(\frac{1}{r^{d}} \exp (-\mu r)\right. \\
& \left.+\frac{1}{\left(N_{\mathrm{s}}-r\right)^{d}} \exp \left[-\mu\left(N_{\mathrm{s}}-r\right)\right]\right),
\end{aligned}
$$

with an arbitrary power $d$ and $\mu$ defines the Debye screening. Because of the tunneling in finite volumes $\mu_{\mathrm{m} . \mathrm{g}}$ is not zero at $\theta>\theta_{\mathrm{c}}$, which influences strongly the functional form of $V_{\mathrm{q} \overline{\mathrm{q}}}(r)$ in the critical region. The parametrization as in eq. (5) is not correct in a finite volume at temperatures near the critical one $\theta \sim \theta_{\mathrm{c}}$.

Monte Carlo studies [4-8] of the heavy quark potential so far gave no arguments in favour of perturbative behaviour at large distances. It is possible that nonperturbative modes play an important role in the large distance behaviour of the chromoplasma even at high temperatures $[9,10]$. Moreover, the very validity of the perturbative approach is now under question. At large enough distances $r$ this expansion is divergent for all (nonzero) couplings $g_{R}^{2}$ and therefore not even the lowest-order calculations are reliable [11]. The determination of the heavy quark potential appears thus to be a nonperturbative problem even at very high temperatures (see, however, refs. $[12,13])$.

As a first step in the investigation of the heavy quark potential we want to deduce the value of the mass gap, $\mu_{\mathrm{m} . \mathrm{g} .}$ (or for $\beta<\beta_{\mathrm{c}}$ the inverse of the corre- lation length $\xi_{-}$) from the full correlator $\Gamma(x)$. To that end we use data from $4 N_{\mathrm{s}}^{3}$ lattices, i.e. with cubic geometry, which were taken in the course of a general finite size scaling analysis [14] with the intention to extract the correlation length and the potential $V_{\mathrm{qq}}(r)$. We shall show that full correlator data may be used just as well as zero momentum correlator data to evaluate the mass gap.

The dependence of the mass gap $\mu_{\mathrm{m} . \mathrm{g}}$ on $\beta$ was already studied for $\mathrm{SU}(2)$ on lattices with cylindrical geometry $N_{\mathrm{t}} \cdot N_{\mathrm{s}}^{2} \cdot N_{z}\left(N_{z} \gg N_{\mathrm{s}}\right)$ [15-17] using zero momentum correlators. The cylindrical geometry has the obvious advantage that the highest excitation level of the transfer matrix may be projected out more reliably than in the cubic case for the same $N_{\mathrm{s}}$. We are aware of this fact and will therefore, when we estimate numbers as the critical coupling and the surface tension, leave the results from our smallest $\left(N_{\mathrm{s}}=8\right)$ lattice out of consideration and additionally check the other results. As we shall see in the following the mass gap and matrix element values which we obtain from our cubic lattices are nevertheless well in accord with the expectations from finite size scaling and universality.

\section{Correlators of Polyakov loops in finite volumes}

We define the zero momentum operators $\widetilde{P}(z)$ as follows:

$\widetilde{P}(z)=\frac{1}{N_{s}} \sum_{x_{\perp}} \mathscr{P}(\boldsymbol{x})$.

In the transfer matrix formalism the zero momentum correlators $\tilde{\Gamma}(z)$ are given by

$$
\begin{aligned}
& \tilde{\Gamma}(z)=\langle\tilde{\mathscr{P}}(z) \cdot \tilde{\mathscr{P}}(0)\rangle \\
& \quad=Z^{-1} \sum_{n, m}\langle n|\tilde{\mathscr{P}}(0)| m\rangle^{2} \\
& \quad \times \exp \left(-\mu_{m} z\right) \exp \left[-\mu_{n}\left(N_{\mathrm{s}}-z\right)\right] \\
& \quad=v^{2}\left\{\exp \left(-z \mu_{\text {m.g. }}\right)+\exp \left[-\left(N_{\mathrm{s}}-z\right) \mu_{\text {m.g. }}\right]\right\} \\
& \quad+v_{1}^{2}\left\{\exp \left(-z \mu_{1}\right)+\exp \left[-\left(N_{\mathrm{s}}-z\right) \mu_{1}\right]\right\}-\ldots,
\end{aligned}
$$

where $v^{2} \equiv Z^{-1}\left\langle 0_{\mathrm{s}}|\tilde{P}(0)| 0_{\mathrm{a}}\right\rangle^{2}$ and $v_{1}, \mu_{1}, \ldots$ correspond to higher exitations of the spectrum of the transfer matrix.

It is worthwhile to note here that in a finite volume 
at $\beta \simeq \beta_{\mathrm{c}}$ rotational invariance is broken: $\Gamma(x, y, z) \equiv$ $\Gamma(\boldsymbol{x}) \neq \Gamma(|\boldsymbol{x}|)$ and only invariance with respect to permutations $x, y, z$ survives. Because in our data Polyakov loops are separated by distance $r$ along one of the three axes we shall use the notation $\Gamma(r) \equiv \Gamma(x)$.

With the definition of the zero momentum operator $\mathscr{P}(z)$ as in eq. (6) the connection between the zero momentum correlator $\tilde{\Gamma}(z)$ and the point-point correlator $\Gamma\left(x_{\perp} ; z\right)$ on a finite lattice is

$\tilde{\Gamma}(z) \equiv\langle\tilde{\mathscr{P}}(z) \tilde{\mathscr{P}}(0)\rangle=\sum_{\boldsymbol{x} \perp} \Gamma\left(\boldsymbol{x}_{\perp} ; z\right)$.

The Fourier-transforms of the correlators $\Gamma(x)$ and $\tilde{\Gamma}(z)$ are

$$
\begin{gathered}
\Gamma(\boldsymbol{p})=\sum_{\boldsymbol{x}} \exp (\mathrm{i} \boldsymbol{x} \cdot \boldsymbol{p}) \Gamma(\boldsymbol{x}), \\
\tilde{\Gamma}\left(p_{z}\right)=\sum_{z} \exp \left(\mathrm{i} z p_{z}\right) \tilde{\Gamma}(z) \\
\equiv \sum_{\boldsymbol{x}} \exp \left(\mathrm{i} z p_{z}\right) \Gamma(\boldsymbol{x})
\end{gathered}
$$

where

$p_{i}=\frac{2 \pi}{N_{\mathrm{s}}} k_{i}, \quad k_{i}=0, \pm 1, \ldots, \pm\left(\frac{1}{2} N_{\mathrm{s}}-1\right), \frac{1}{2} N_{\mathrm{s}}$

for even values of $N_{\mathrm{s}}$. Therefore the correlators $\Gamma$ and $\tilde{\Gamma}$ are connected by the evident relation

$\tilde{\Gamma}\left(p_{z}\right) \equiv \Gamma\left(\boldsymbol{p}_{\perp}=0, p_{z}\right)$.

Discarding higher exitations in eq. (7) $\left(\mu=\mu_{\text {m.g. }}\right)$ one obtains for the Fourier-transform $\tilde{\Gamma}\left(p_{z}\right)$

$\tilde{\Gamma}\left(p_{z}\right)=v^{2} \cdot 2 \mu\left[1-\exp \left(-N_{\mathrm{s}} \mu\right)\right] G\left(p_{z} ; N_{\mathrm{s}}, \mu\right)$,

where

$$
\begin{aligned}
& G\left(p_{z} ; N_{\mathrm{s}}, \mu\right) \equiv \frac{1-\exp (-2 \mu)}{2 \mu}\left\{[1-\exp (-\mu)]^{2}\right. \\
& \left.\quad+\exp (-\mu) \cdot 4 \sin ^{2}\left(\frac{1}{2} p_{z}\right)\right\}^{-1} .
\end{aligned}
$$

To derive the correlator $\Gamma(\boldsymbol{p})$ we use the following substitution in eq. (14):

$4 \sin ^{2}\left(\frac{1}{2} p_{z}\right) \rightarrow D(p) \equiv \sum_{i=1}^{3} 4 \sin ^{2}\left(\frac{1}{2} p_{i}\right)$,

where $D(\boldsymbol{p})$ is just the lattice laplacian in 3D momentum space. One cannot exclude in principle that in the denominator of $G(\boldsymbol{p} ; N, \mu)$, say, cross-terms $\sim$ $\sin ^{2}\left(\frac{1}{2} p_{i}\right) \sin ^{2}\left(\frac{1}{2} p_{i}\right)$ may appear. So, our ansatz eq. (15) is based on the assumption that these crossterms play a negligible (if any) role. As a result we find

$$
\Gamma(\boldsymbol{p})=v^{2} \cdot 2 \mu\left[1-\exp \left(-N_{\mathrm{s}} \mu\right)\right] G\left(\boldsymbol{p} ; N_{\mathrm{s}}, \mu\right),
$$

where

$$
\begin{aligned}
& G\left(\boldsymbol{p} ; N_{\mathrm{s}}, \mu\right) \equiv \frac{1-\exp (-2 \mu)}{2 \mu}\left([1-\exp (-\mu)]^{2}\right. \\
& \left.\quad+\exp (-\mu) \sum_{i=1}^{3} 4 \sin ^{2}\left(\frac{1}{2} p_{i}\right)\right)^{-1} .
\end{aligned}
$$

Performing the inverse Fourier-transform one arrives at the following expression for the correlator $\Gamma(r)$ :

$$
\begin{aligned}
& \Gamma(r)=v^{2} \cdot 2 \mu\left[1-\exp \left(-N_{\mathrm{s}} \mu\right)\right] \frac{1}{N_{\mathrm{s}}^{3}} \\
& \quad \times \sum_{\boldsymbol{p}} \exp \left(-\mathrm{i} r p_{z}\right) G\left(\boldsymbol{p}=\frac{2 \pi}{N_{\mathrm{s}}} \boldsymbol{k} ; N_{\mathrm{s}}, \mu\right) .
\end{aligned}
$$

In the limit of small $\mu\left(\mu \ll N_{\mathrm{s}}^{-1}\right)$ the correlator $\Gamma(r)$ will tend to a constant value. At $\mu N_{\mathrm{s}} \gg 1$ the correlator $\Gamma(r)$ is equivalent to the superposition of two $\mathrm{Yu}-$ kawa-type potentials:

$$
\begin{aligned}
& \Gamma_{\text {Yuk }}(r) \equiv v^{2} \frac{\mu}{2 \pi}\left[1-\exp \left(-\mu N_{\mathrm{s}}\right)\right] \\
& \quad \times\left(\frac{1}{r} \exp (-\mu r)+\frac{1}{N_{\mathrm{s}}-r} \exp \left[-\mu\left(N_{\mathrm{s}}-r\right)\right]\right) .
\end{aligned}
$$

Fig. 1 shows the behaviour of the ratio $R(r) \equiv$ $\Gamma(r) / \Gamma(r)_{\mathrm{Yuk}}$ as a function of $r$ at different values of $\mu N_{\mathrm{s}}$. We observe that $R(r) \simeq 1$ only for $\mu \gg N_{\mathrm{s}}^{-1}$ and, therefore, the correlator $\Gamma(r)$ can be represented in the form of a superposition of two Yukawa-type potentials only far from the phase transition point. At $\beta \sim \beta_{\mathrm{c}}$, where $\mu \sim N_{\mathrm{s}}^{-1}$ finite-volume corrections are too strong and this is not possible.

Defining the average of the "squared magnetization".

$$
\left\langle\mathscr{P}^{2}\right\rangle \equiv \frac{1}{N_{\mathrm{s}}^{3}} \sum_{\boldsymbol{x}} \Gamma(\boldsymbol{x})=\left\langle\left(\frac{1}{N_{\mathrm{s}}^{3}} \sum_{\boldsymbol{x}} \mathscr{P}(\boldsymbol{x})\right)^{2}\right\rangle,
$$

we get for $\left\langle\mathscr{P}^{2}\right\rangle$ from eq. (18) 


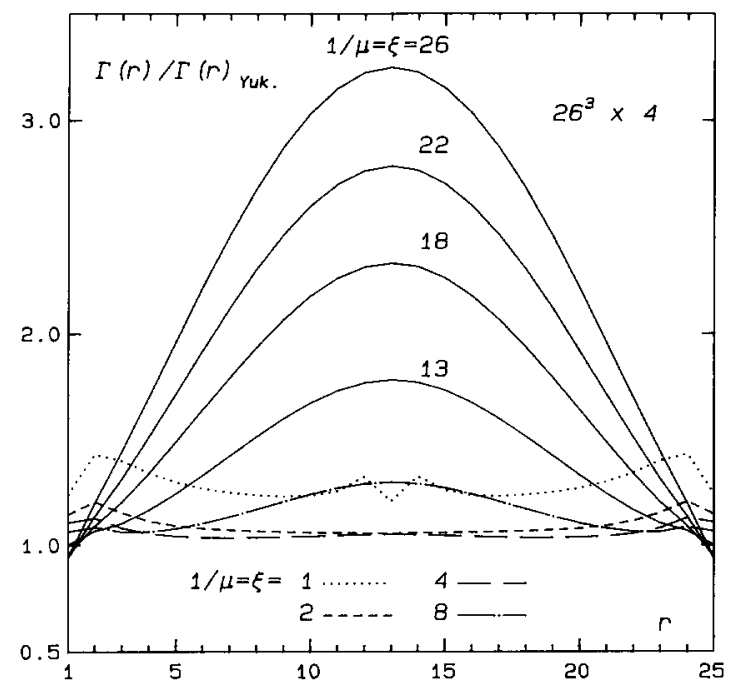

Fig. 1. The behaviour of the ratio $\Gamma(r) / \Gamma(r)_{\mathrm{Yuk}}$ as a function of $r$ at different values of $\mu$ on an $N_{\mathrm{s}}=26$ lattice.

$$
\begin{aligned}
& N_{\mathrm{s}}^{3}\left\langle\mathscr{P}^{2}\right\rangle=v^{2}\left[1-\exp \left(-\mu_{\text {m.g. }} N_{\mathrm{s}}\right)\right] \\
& \quad \times \frac{1+\exp \left(-\mu_{\text {m.g. }}\right)}{1-\exp \left(-\mu_{\text {m.g. }}\right)} .
\end{aligned}
$$

It is worthwhile to note here the nontrivial role of the factor with the explicit size dependence $1-\exp \left(-\mu_{\text {m.g. }} N_{\mathrm{s}}\right)$ in eq. (21). Far below the transition point it tends to unity with increasing lattice size $N_{\mathrm{s}}$. But at $\beta>\beta_{\mathrm{c}}$, where $\mu_{\text {m.g. }} N_{\mathrm{s}} \ll 1$ it gives after contraction with $\mu_{\text {m.g. }}$ from the denominator an additional power of $N_{\mathrm{s}}$.

In the thermodynamic limit below the phase transition point, $\beta<\beta_{\mathrm{c}}$, the correlator $\Gamma(\boldsymbol{x})$ decays exponentially at large distances,

$\Gamma(\boldsymbol{x}) \sim \exp \left(-\frac{r}{\xi_{-}(\beta)}\right), \quad N_{\mathrm{s}} \rightarrow \infty, \quad r \gg 1$,

which entails

$\sum_{\boldsymbol{x}_{\perp}} \Gamma\left(\boldsymbol{x}_{\perp} ; z\right)<\infty$.

From eq. (23) we conclude that for a finite lattice size all matrix elements are independent of $N_{\mathrm{s}}$ in the large volume limit so that

$v^{2} \sim N_{\mathrm{s}}^{0}, \quad N_{\mathrm{s}} \rightarrow \infty$.

Above the transition point, $\beta>\beta_{\mathrm{c}}$, the large distance behaviour of the correlator $\Gamma(x)$ in the thermodynamic limit is

$$
\begin{gathered}
\Gamma(\boldsymbol{x})-\left\langle\mathscr{P}^{2}\right\rangle \sim \exp \left(-\frac{r}{\xi_{+}(\beta)}\right), \\
N_{\mathrm{s} \rightarrow \infty,} \quad r \gg 1,
\end{gathered}
$$

and at large but finite $N_{\mathrm{s}}$

$\sum_{x_{\perp}} \Gamma\left(\boldsymbol{x}_{\perp} ; z\right) \sim N_{\mathrm{s}}^{2}$.

The matrix element has therefore the following dependence on the lattice size:

$v^{2} \sim N_{\mathrm{s}}^{2}, N_{\mathrm{s}} \rightarrow \infty$.

\section{Data analysis}

The data we analyse were produced on lattices with $N_{\mathrm{t}}=4$ and $N_{\mathrm{s}}=8,12,18,26$. Part of the data were already evaluated and described in ref. [14]. The correlators $\Gamma(r)$ were measured every 10 th update in these runs, so that between 10000 and, close to $\theta_{c}$, upto 45000 measurements per $\beta$-value were available.

To extract values of the mass gap $\mu_{\text {m.g. }}\left(\beta ; N_{\mathrm{s}}\right)$ and the matrix element $v\left(\beta ; N_{\mathrm{s}}\right)$ we made simultaneous fits of our data for the correlators $\Gamma(r)$ and the squared magnetization $\left\langle\mathscr{P}^{2}\right\rangle$ using eq. (18) and eq. (21). The errors of $\mu_{\mathrm{m} . \mathrm{g}}$ and the matrix element $v$ were determined from the $\chi^{2}$ of the fits such as to include possible deviations with a probability of $75 \%$. Systematic errors due to the neglect of the higher levels have not been taken into account. Exploratory fits including more levels in formula (18) show that the mass gap values resulting from the fit close to and above the critical point are changed to slightly lower values. A corresponding effect is found if we discard in the fitting procedure the $r=1$ or the $r=1$ and 2 correlator data. Then $\mu_{\mathrm{m} . \mathrm{g}}$-values near to $\beta_{\mathrm{c}}$ are lowered by about $10 \%(12 \%)$ for the bigger lattices and by $40 \%(60 \%)$ for the $N_{\mathrm{s}}=8$ lattice. In general, however, we find from our full correlator formula, eq. (18), mass gap values which are of comparable size to those deduced from zero momentum correlators (see, e.g. ref. [17]).

The dependence of $\mu_{\text {m.g. }}\left(\beta ; N_{\mathrm{s}}\right)$ on $\beta$ for different $N_{\mathrm{s}}$ is shown in fig. 2a. For $\beta \gtrsim 2.27 \mu_{\text {m.g. }}$ it shows strong 

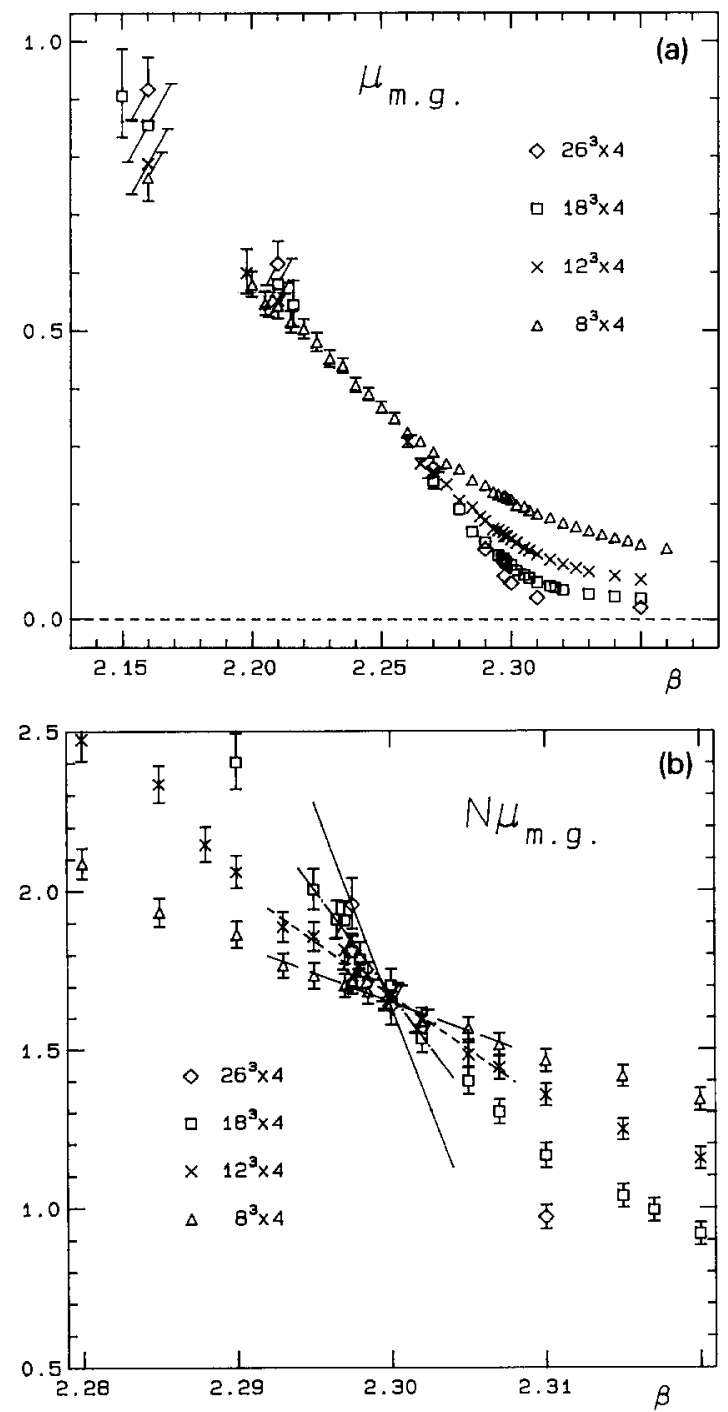

Fig. 2. (a) The dependence of $\mu_{\text {m.g. }}$ on $\beta$ for different $N_{\mathrm{s}}$. (b) The dependence of $N_{\mathrm{s}} \mu_{\mathrm{m} . \mathrm{g}}$ on $\beta$ for different $N_{\mathrm{s}}$ near the transition point $\beta_{\mathrm{c}}$. The straight lines are linear least square fits to the points in the neighbourhood of $\beta_{\mathrm{c}}$.

finite-volume effects as expected. Also the mass gap is tending to zero with increasing $N_{\mathrm{s}}$, as it should be near to and above the critical point.

To scrutinize the temperature and finite-volume dependence of the mass gap we used the finite-size scaling (FSS) technique $[18,19]$. Below the critical point the mass gap can be identified with the inverse correlation length (see, e.g. ref. [19]): $\xi_{-}\left(\beta ; N_{\mathrm{s}}\right) \equiv \mu_{\mathrm{m} \cdot \mathrm{g} .}^{-1}\left(\beta ; N_{\mathrm{s}}\right), \quad \beta \leqslant \beta_{\mathrm{c}}$.

According to FSS theory any observable $O$ with critical behaviour is supposed to have the following form;

$O=N_{\mathrm{s}}^{\rho / v} f_{O}\left(x N_{\mathrm{s}}^{1 / \nu}\right), \quad N_{\mathrm{s}} \rightarrow \infty$,

for fixed small $x \equiv\left(\beta-\beta_{\mathrm{c}}\right) / \beta_{\mathrm{c}}$ and $\rho$ is the critical exponent of the observable $O$. Since the critical exponent of the correlation length is again $\nu$ we expect for the mass gap

$\mu_{\text {m.g. }}(\beta ; N)_{\mathrm{s}}=\frac{1}{N_{\mathrm{s}}} f_{\mu}\left(x N_{\mathrm{s}}^{1 / \nu}\right), \quad x \sim 0$

and at the critical point

$\mu_{\mathrm{m} . \mathrm{g} .}\left(\beta_{\mathrm{c}} ; N_{\mathrm{s}}\right) \sim N_{\mathrm{s}}^{-1}$,

$\frac{\mathrm{d}}{\mathrm{d} \beta} \mu_{\mathrm{m} . \mathrm{g} .}\left(\beta_{\mathrm{c}} ; N_{\mathrm{s}}\right) \sim N_{\mathrm{s}}^{-1+1 / \nu}$.

Below the transition point the susceptibility $\chi(\beta$; $N_{\mathrm{s}}$ ) is defined as follows:

$\chi\left(\beta ; N_{\mathrm{s}}\right)=N_{\mathrm{s}}^{3}\left\langle\mathscr{P}^{2}\right\rangle, \quad \beta \leqslant \beta_{\mathrm{c}}$.

The critical exponent of the susceptibility is $\gamma$ and eq. (29) then leads to

$\left\langle\mathscr{P}^{2}\right\rangle=N_{\mathrm{s}}^{-3+\gamma / \nu} f_{\mathscr{P}}\left(x N_{\mathrm{s}}^{1 / \nu}\right), \quad x \sim 0$.

Combining eqs. (21), (30) and (34) we finally obtain

$v^{2}(\beta)=N_{\mathrm{s}}^{-1+\gamma / \nu} f_{v}\left(x N_{\mathrm{s}}^{1 / \nu}\right), \quad x \sim 0$.

The following should be mentioned here. Eq. (28) and eq. (33) are valid only at $\beta \leqslant \beta_{c}(x \leqslant 0)$. Nevertheless, because of the analytic dependence on $\beta$ (or $x$ ) on a finite lattice we expect that the corresponding FSS equations ( 30$),(34)$ and (35) will be valid also above the critical point, i.e., at $x \gtrsim 0$. This is really the case as can be seen below.

We used eq. (31) to estimate the transition point $\beta_{\mathrm{c}}$. Though observables, which are directly connected to the measured data (i.e. not through a fit) like the cumulant $g_{r}$ (see ref. [14]) or $\left\langle\mathscr{P}^{2}\right\rangle$ are better suited to that purpose, this gives us a check on the consistency of our results for the mass gap and a comparison to the zero momentum results. In fig. $2 b$ we show the dependence of $N_{\mathrm{s}} \mu_{\mathrm{m} . \mathrm{g}}$. on $\beta$ for different values of $N_{\mathrm{s}}$ in a narrow region near $\beta_{\mathrm{c}}$. The straight lines cor- 
respond to linear least square fits of $N_{\mathrm{s}} \mu_{\mathrm{m} . \mathrm{g} .}$ near the transition point. We have chosen the intervals where these fits were done much smaller (by a factor three or more) than in ref. [17]. This was necessary because, as can be seen in fig. $2 \mathrm{~b}$, with increasing $N_{\mathrm{s}}$ the curvature of $N_{\mathrm{s}} \mu_{\mathrm{m} . \mathrm{g}}$ increases and it was possible since we have enough points close to the critical point.

The intersection point $\beta\left(N_{\mathrm{s}}, N_{\mathrm{s}}^{\prime}\right)$ of the two lines corresponding to the lattice sizes $N_{\mathrm{s}}$ and $N_{\mathrm{s}}^{\prime}$ gives an estimate of the critical point. At large enough values of $N_{\mathrm{s}}, N_{\mathrm{s}}^{\prime}$ the intersection point must tend to $\beta_{\mathrm{c}}$. We obtain for $\beta\left(N_{\mathrm{s}}, N_{\mathrm{s}}^{\prime}\right)$

$$
\begin{aligned}
& \beta(12,8)=2.3013 \pm 0.0012, \\
& \beta(18,8)=2.3005 \pm 0.0006, \\
& \beta(18,12)=2.3001 \pm 0.0009, \\
& \beta(26,8)=2.2999 \pm 0.0006, \\
& \beta(26,12)=2.2997 \pm 0.0006, \\
& \beta(26,18)=2.2994 \pm 0.0009 .
\end{aligned}
$$

With increasing, $N_{\mathrm{s}}, N_{\mathrm{s}}^{\prime}$ the intersection point $\beta\left(N_{\mathrm{s}}\right.$, $N_{\mathrm{s}}^{\prime}$ ) approaches the critical point from above, and $\beta(26,18)$ in eq. $(36)$ is in agreement with the value obtained in ref. [14], $\beta_{\mathrm{c}}=2.2985 \pm 0.0006$. The slight discrepancy with the value $\beta_{\mathrm{c}}$ obtained in ref. [17] can be naturally explained by the fact that the lattice volumes used in that paper are not large enough.

Given the critical point $\beta_{\mathrm{c}}$ one can determine the critical exponent $\nu$ using eq. (32). Fig. 3 shows the dependence of $\ln \left(-\mathrm{d} N_{\mathrm{s}} \mu_{\mathrm{m} . \mathrm{g} .} / \mathrm{d} \beta\right)$ on $\ln N_{\mathrm{s}}$ at $\beta=\beta_{\mathrm{c}}$. The straight line is a linear least square fit of our data with the inverse slope

$\nu=0.62 \pm 0.08$.

The error bars in eq. (37) are comparatively large, but the value of $\nu$ obtained agrees well with $\nu_{\text {Ising }} \simeq$ 0.63 for the 3D Ising model (indicated by the dashed line in fig. 3 ).

To demonstrate the consistency of our data with the universality hypothesis [20], which predicts equal critical exponents for $\mathrm{SU}(2)$ and the 3D Ising model, we show in fig. $4 \mathrm{a} N_{\mathrm{s}} \mu_{\mathrm{m} . \mathrm{g} \text {. }}$ and in fig. $4 \mathrm{~b} v^{2} N_{\mathrm{s}}^{-1+\gamma / \nu}$ as a function of $y \equiv x N_{\mathrm{s}}^{1 / \nu}$, where $\nu$ and $\gamma$ were taken from the 3D Ising model. We see that within the error bars all data points in the vicinity of the phase transition lie on the same universal curve, i.e. we have scaling.

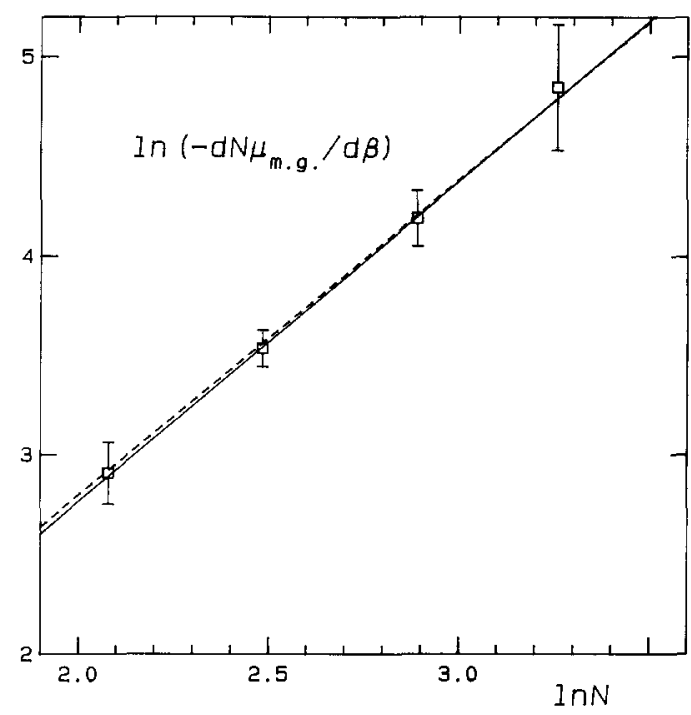

Fig. 3. The dependence of $\ln \left(-\mathrm{d} N_{\mathrm{s}} \mu_{\mathrm{m} . \mathrm{g} .}\left(\beta ; N_{\mathrm{s}}\right) / \mathrm{d} \beta\right)$ on $\ln N_{\mathrm{s}}$ at $\beta=\beta_{\mathrm{c}}$. The line is a linear least square fit to the data, the dashed one corresponds to a fit with $\nu=0.63$ as input.

Using our values for $\nu$ from eq. (37) and $\beta_{\mathrm{c}}=\beta(26$, 18 ) from eq. (36) leads to plots which cannot be distinguished by eye from fig. 4 .

The tunneling in finite volumes can be interpreted as the creation of interfaces between domains with different signs of "spins" $\mathscr{P}(\boldsymbol{x})$ separated by domain walls. The associated interface energy density (surface tension ) $\alpha_{\text {s.t. }}$ is defined as

$\theta \alpha_{\mathrm{s} . \mathrm{t} .}(\theta)=\frac{\mathscr{F}_{\mathrm{tw}}-\mathscr{F}}{N_{x} N_{y}} \equiv \frac{\mathscr{F}_{\mathrm{tw}}-\mathscr{\mathscr { F }}}{N_{\mathrm{s}}^{2}}$,

where the free energies $\mathscr{F}$ and $\mathscr{F}_{\text {tw }}$ are defined as

$\mathscr{F}(\theta)=-\theta \ln Z, \quad \mathscr{F}_{\mathrm{tw}}(\theta)=-\theta \ln Z_{\mathrm{tw}}$,

and $Z_{\mathrm{tw}}$ is the partition function on the lattice with twisted boundary conditions in the $(t, z)$-plane $[21,22]$. The surface tension is connected to the mass gap $\mu_{\text {m.g. }}$ through the following relation:

$\mu_{\text {m.g. }} \sim \exp \left[-\alpha_{\text {s.t. }}(\theta) N_{\mathrm{s}}^{2}\right]$,

where the preexponential factor depends on temperature and can comprise some power dependence $\sim N_{\mathrm{s}}^{b}$. One may speculate, referring to the analogy with the $3 \mathrm{D}$ Ising model, that $b=0[23,24]$. In fig. 5 we show the dependence of $-\ln \mu_{\text {m.g. }}(\theta) / N_{\mathrm{s}}^{2}$ on $1 /$ $N_{\mathrm{s}}^{2}$ at $\beta=2.35$ for $N_{\mathrm{s}}=12,18,26$. To make sure that 

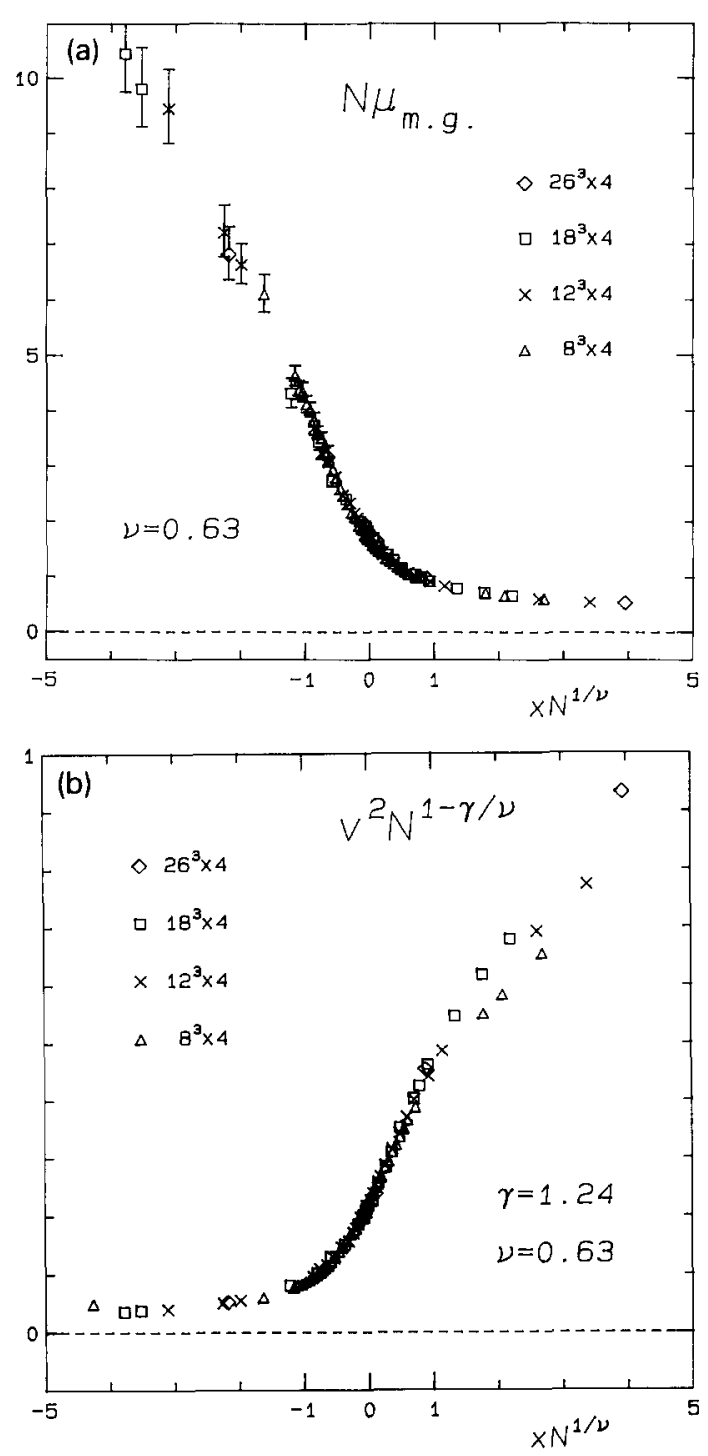

Fig. 4. (a) The dependence of $N_{\mathrm{s}} \mu_{\text {m.g. }}\left(\beta ; N_{\mathrm{s}}\right)$ on $x N_{\mathrm{s}}^{1 / \nu}$ $x \equiv\left(\beta-\beta_{\mathrm{c}}\right) / \beta_{\mathrm{c}}$. (b) The dependence of $v^{2} N_{\mathrm{s}}^{1-\gamma / \nu}$ on $x N_{\mathrm{s}}^{1 / \nu}$, with the 3D Ising model values for $\gamma$ and $\nu$ as input.

our values for $\mu_{\text {m.g. }}$ are as realistic as possible, we have made several fits to the correlator data: including all distances, omitting distance $r=1$ and $r=1$ and 2. The points shown in fig. 5 correspond to the second of these fits. There is essentially no difference to the third fit and only a slight one to the first. The broken line in the figure is a linear least square fit to our data with the ansatz $\mu_{\text {m.g. }}=c_{\alpha} \exp \left(-\alpha_{\text {s.t. }} N_{\mathrm{s}}^{2}\right)$, where

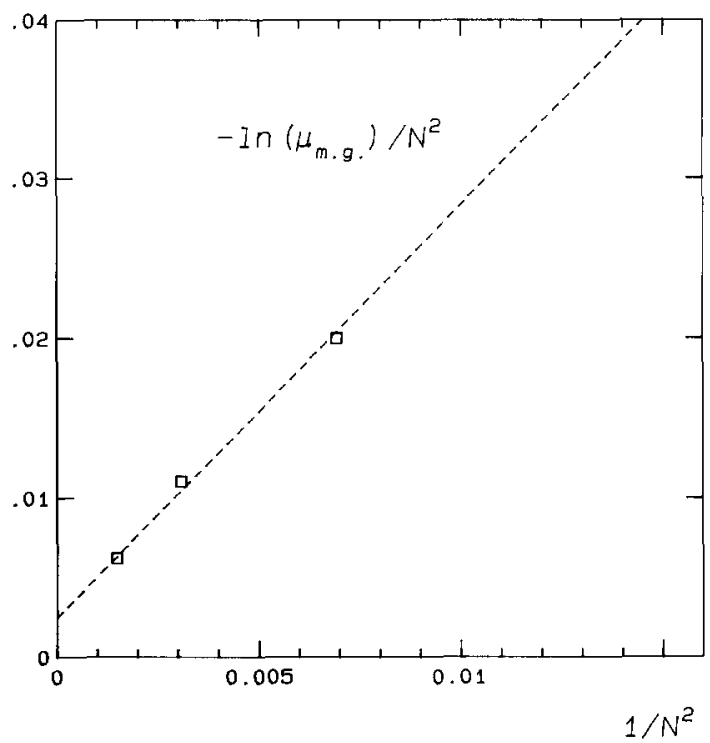

Fig. 5. The dependence of $-\ln \mu_{\mathrm{m} . \mathrm{g}}\left(\beta ; N_{\mathrm{s}}\right) / N_{\mathrm{s}}^{2}$ on $1 / N_{\mathrm{s}}^{2}$ at $\beta=2.35$. The broken line is a linear least square fit to the data for our three largest lattice volumes.

$\alpha_{\text {s.t. }}(\beta=2.35)=0.0025(2)$ and $c_{\alpha}=0.075(5)$. Inside the error bars the surface tension obtained from respective linear fits to the other two sets of data is compatible with this result. A two level fit to the correlator data also leads to the same $\alpha_{\text {s.t. }}$-value.

\section{Conclusions}

In this paper we studied the point-point Polyakov loop correlators $\Gamma(r)$ in SU(2) lattice gauge theory on $4 N_{\mathrm{s}}^{3}$ lattices with $N_{\mathrm{s}}=8,12,18$ and 26. In particular we were interested in the role of finite volume effects near the transition point $\beta_{\mathrm{c}}$.

Our analysis is based on an analytic expression for the point-point correlator provided by the transfer matrix formalism and the ansatz in eq. (15). The results of our analysis testify in favour of its validity.

We studied the $\beta$ (temperature) dependence of the mass gap $\mu_{\text {m.g. }}$ and the corresponding matrix element $v$ near the critical point in a finite volume. The finitesize scaling analysis of the values obtained from the fit allows to determine the critical value $\beta_{\mathrm{c}}$, the critical exponent $\nu$, as well as the surface tension $\alpha_{\text {s.t. }}$. The value of the critical point $\beta$ which we obtained is in 
agreement with the results from the analysis of the cumulant [14] and of the zero momentum correlators [17]. The value of the critical exponent $\nu$ is consistent with that of the 3D Ising model.

In this paper we did not consider higher-order contributions to the correlators $\Gamma(r)$ and the "squared magnetization" $\left\langle\mathscr{P}^{2}\right\rangle$. They can produce some bias of the values of the mass gap but not for the value of the transition point and the critical exponents. The study of the temperature and finite volume dependence of these higher-order contributions is a nontrivial but very interesting problem especially important for the investigation of the heavy quark potential. This will be a main topic of a forthcoming paper.

\section{Acknowledgement}

We would like to thank B. Berg, L. Kärkkäinen and T. Neuhaus for useful discussions. We are indepted to J. Fingberg and M. Weber for their help in the measurement of the correlation data. Also one of us (V.K.M.) would like to express his gratitude to Bielefeld University for hospitality.

\section{References}

[1] A.M. Polyakov, Phys. Lett. B 72 (1978) 477.

[2] L. Susskind, Phys. Rev. D 20 (1979) 2610.

[3] C. Gale and J. Kapusta, Phys. Lett. B 198 (1987) 89.

[4] Ph. De Forcrand, G. Schierholz, H. Schneider and M. Teper, Phys. Lett. B 160 (1985) 137.
[5] K. Kanaya and H. Satz, Phys. Rev. D 34 (1986) 3193.

[6] T.A. DeGrand and C.E. DeTar, Phys. Rev. D 34 (1986) 2469.

[7] N. Attig, F. Karsch, B. Petersson, H. Satz and M. Wolff, Phys. Lett. B 209 (1988) 65.

[8] J. Engels, F. Karsch and H. Satz, Nucl. Phys. B 315 (1989) 419.

[9] E. Manousakis and J. Polonyi, Phys. Rev. Lett. 58 (1987) 847.

[10] J.B. Kogut and C.A. De Tar, Phys. Rev. D 36 (1987) 2828.

[11] S. Nadkarni, Phys. Rev. D 33 (1986) 3738; D 34 (1986) 3904; Physica A 158 (1989) 226.

[12] B. Petersson and T. Reisz, Nucl. Phys. B 353 (1991) 757.

[13] A. Irbäck, P. LaCock, D. Miller, B. Petersson and T. Reisz, Nucl. Phys. B 363 (1991) 34.

[14] J. Engels, J. Fingberg and M. Weber, Nucl. Phys. B 332 (1990) 737.

[15] B.A. Berg, A.H. Billoire and R. Salvador, Phys. Rev. D 37 (1988) 3774 .

[16] B.A. Berg and A.H. Billoire, Phys. Rev. D 40 (1989) 550.

[17] B.A. Berg, R. Villanova and C. Vohwinkel, Phys. Rev. Lett. 62 ( 1989$) 2433$.

[18] M.E. Fisher, in: Critical phenomena, Proc. 51 st Enrico Fermi Summer School, (Varenna, Italy), ed. M.S. Green (Academic Press, New York, 1971).

[19] M.N. Barber, in: Phase transitions and critical phenomena, Vol. 8, eds. C. Domb and J.L. Lebovitz (Academic Press, New York, 1983) p. 146.

[20] B. Svetitsky and G. Yaffe, Nucl. Phys. B 210 [FS6] (1982) 423.

[21 ] G. 't Hooft, Nucl. Phys. B 153 (1979) 141.

[22] J. Groeneveld, J. Jurkiewicz and C.P. Kortals Altes, Phys. Scr. 23 (1981) 1022.

[23] E. Brézin and J. Zinn-Justin, Nucl. Phys. B 257 (1985) 867.

[24] C. Borgs and J.Z. Imbrie, Harvard preprint 91-0233. 